\title{
MicroRNA-203 Acts as a Potent Suppressor in Septic Shock by Alleviating Lung Injury via Inhibition of VNN1
}

\author{
Lan Ling $^{\mathrm{a}}$ Hai-Tao Lu ${ }^{\mathrm{b}}$ Hai-Feng Wang ${ }^{\mathrm{b}}$ Mei-Jia Shen ${ }^{\mathrm{c}}$ Hong-Bo Zhang ${ }^{\mathrm{a}}$ \\ aEmergency Department, China-Japan Friendship Hospital, Beijing, China; ${ }^{b}$ Nephropathy \\ Department, China-Japan Friendship Hospital, Beijing, China; ${ }^{c}$ Clinical Institute, China-Japan \\ Friendship Hospital, Beijing, China
}

\section{Keywords}

MicroRNA-203 · Septic shock · Vanin-1 · AKT signaling pathway · Lung injury · Oxidative stress - Apoptosis

\begin{abstract}
Background: Septic shock, the most serious complication of sepsis, is a life-threatening disease that is mainly characterized by hypoperfusion and multiple organ failure. Various aberrantly expressed microRNAs (miRNAs) have been reported to be related to septic shock. We explored the regulatory effect of microRNA-203 (miR-203) on lung injury in septic shock mice. Methods: Microarray-based gene expression profiling related to septic shock identified the differentially expressed gene vanin-1 (VNN1) and potential regulatory miR-203. miR-203 was predicted to mediate VNN1 expression, thus affecting septic shock, which was investigated by treatment with miR-203 mimic, miR-203 inhibitor, and siRNA-VNN1 in septic shock mouse models. Polymorphonuclear neutrophils (PMNs) and pulmonary alveolar macrophages in bronchoalveolar lavage fluid (BALF) as well as the wet/dry ratio of the lung were also measured to assess lung injury. Additionally, the effects of miR-203 on inflammatory cytokines, oxidative stress indexes, blood biochemical indexes, serine-threonine protein kinase (AKT) signaling pathway-related factors, and apoptosis-related factors were determined. Results: VNN1 was verified to be targeted and negatively regulated by miR-203. In mouse models of septic shock, weak expression of miR-203, high expression of VNN1, and inhibition of AKT signaling pathway were identified. In response to miR-203 mimic and VNN1 gene silencing, mouse models of septic shock displayed reduced apoptosis, MDA, ALT, and AST in lung tissues, decreased levels of TNF- $\alpha$, IL-1 $\beta$, IFN- $\gamma$, IL-10, and IL- 6 , in serum, and reduced PMN and
\end{abstract}




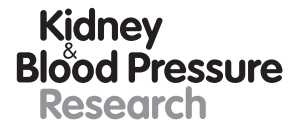

Kidney
Blood Pressure
Research

\begin{tabular}{l|l}
\hline Kidney Blood Press Res 2019;44:565-582 \\
\hline DOI: 10.1159/000500484 & $\begin{array}{l}\text { @ 2019 The Author(s). Published by S. Karger AG, Basel } \\
\text { www.karger.com/kbr }\end{array}$ \\
\hline
\end{tabular}

Ling et al.: Role of miR-203 in Septic Shock

PAM levels in BALF, in addition to elevated SOD activity. Notably, the presence of miR-203 mimic led to AKT signaling pathway activation. Conclusion: This study shows that upregulating miR-203 can alleviate lung injury through activation of the AKT signaling pathway by downregulating VNN1 in septic shock.

\section{Introduction}

Septic shock is a prevalent complication in the intensive care unit, and it is also closely related to public health [1]. As a systemic inflammatory response to infection, the pathophysiological abnormalities of septic shock are characterized by cardiovascular dysfunction, neutrophil activation, and infiltration into multiple organs [2]. Due to an imbalance of the innate immune response after infection, septic shock is associated with a $50 \%$ morality rate despite significant advances achieved in basic and clinical research [3]. The death of patients with severe sepsis mainly results from multiple organ dysfunction, among which the respiratory system is the most commonly involved organ, and nearly half of patients with severe sepsis will develop acute lung injury [4]. In the treatment of septic shock, the common process is rescue, optimization, stabilization, and de-escalation of care, and adults often immediately receive intravenous injection, liquid injection, vasopressors, and care to restore sufficient circulation [5]. Additionally, according to a recent study, 13 microRNAs (miRNAs) participate in the regulation of septic shock, and there is a connection between the levels of these miRNAs and organ failure resulting from septic shock [6].

Recently, miRNAs with a length of approximately 19-22 nucleotides have become a key focus of biomedical research since these small noncoding molecules posttranscriptionally mediate gene expression in various biological and pathological processes [7]. Diverse aberrantly expressed miRNAs have been reported to contribute to septic shock, acute lung injury, and inflammatory responses $[8,9]$. miRNAs are related to cell invasion, apoptosis, and proliferation [10]. A low expression level of miR-203 was previously found to be an indicator of non-small-cell lung cancer [11]. Vanin-1 (VNN1), located on chromosome 6q23-q24, is a glycosylphosphatidylinositol-anchored pantetheinase and shows a high expression level in the liver and kidney $[12,13]$. A serine-threonine protein kinase (AKT), also named protein kinase B, refers to an antiapoptotic protein kinase in mammals [14]. An association between VNN1 and AKT indicates that overexpressed VNN1 decreases the extent of AKT phosphorylation [15]. Moreover, miR-203 is able to activate the AKT signaling pathway through interleukin (IL)-8 in the regulation of nasopharyngeal carcinoma cell radioresistance [16]. Additionally, the AKT signaling pathway has been demonstrated to be effective in preventing ventilator-induced lung injury [17]. In this study, we will explore the regulatory effect of miR-203 on lung injury in septic shock with involvement of the AKT signaling pathway and VNN1 to elucidate a theoretical basis for further treatment of septic shock.

\section{Materials and Methods}

\section{Microarray Analysis}

The gene chip for septic shock was obtained from Gene expression Omnibus (GEO) database of the National Center for Biotechnology Information (https://www.ncbi.nlm.nih. gov/). Six datasets, GSE95233, GSE570655, GSE8121, GSE4607, GSE26440, and GSE26378, were used for differentially expressed gene (DEG) screening. The details of the gene anno- 


\section{Kidney \\ Blood Pressure \\ Research}

\begin{tabular}{l|l}
\hline Kidney Blood Press Res 2019;44:565-582 \\
\hline DOI: 10.1159/000500484 & $\begin{array}{l}\text { @ 2019 The Author(s). Published by S. Karger AG, Basel } \\
\text { www.karger.com/kbr }\end{array}$ \\
\hline
\end{tabular}

Ling et al.: Role of miR-203 in Septic Shock

Table 1. Information on septic shock-related datasets

\begin{tabular}{lllll}
\hline Accession & Platform & Organism & Tissue & Sample \\
\hline GSE95233 & GPL570 & Homo sapiens & Blood & 20 septic shock patients and 22 healthy volunteers \\
GSE57065 & GPL570 & Homo sapiens & Whole blood & 26 septic shock patients and 25 healthy volunteers \\
GSE8121 & GPL570 & Homo sapiens & Whole blood & 15 normal and 30 septic shock children \\
GSE4607 & GPL570 & Homo sapiens & Whole blood & 15 controls, 42 children with septic shock \\
GSE26440 & GPL570 & Homo sapiens & Whole blood & 98 children with septic shock and 32 normal controls \\
GSE26378 & GPL570 & Homo sapiens & Whole blood & 82 children with septic shock and 21 normal controls \\
\hline
\end{tabular}

tation platforms and samples of the datasets are shown in Table 1. In R language, the limma package (http://master.bioconductor.org/packages/release/bioc/html/limma.html) was used to identify the DEGs between septic shock samples and normal controls. The log2-fold change $(\log 2 \mathrm{FC})$ was calculated. The screening conditions for the DEGs were set as $|\log 2 \mathrm{FC}|$ $>2.0$ and $p<0.05$, and the heat map of the DEGs was plotted. Jvenn (http://jvenn.toulouse. inra.fr/app/example.html) can compare and analyze different combinations of elements and visualize the results to draw a Venn diagram [18], which was used to compare the DEGs in six chips. The DisGeNET Gene-Disease Relation Database (http://www.disgenet.org/web/ DisGeNET/menu/search?4) was employed for genes related to septic shock. The interaction between DEGs of septic shock and disease-related genes was analyzed using the String database (https://string-db.org/), and the interaction network was visualized using Cytoscape 3.6.0 software [19]. TargetScan (http://www.targetscan.org/vert_71/) and microRNA (http://34.236.212.39/microrna/getGeneForm.do) were used to predict the target miRNAs of the DEGs, and then jvenn was used to compare the results for the miRNA prediction.

\section{Model Establishment}

Sixty-eight normal clean grade Kunming mice were selected (weight: approximately 25-30 g; half male and half female; Department of Laboratory Animal Sciences, Shanghai Jiao Tong University, Shanghai, China). Forty-nine mice were randomly selected for the cecal ligation and puncture (CLP) group. After anesthesia with ether, the hair of the right abdomen was shaved, and a $0.5-\mathrm{cm}$ longitudinal incision was created along the middle right of the abdominal line to expose the cecum, small intestine and colon of the mice. The cecum was ligated at the base, and then perforated in two locations with a disinfected 18-gauge needle distal to the point of ligation. Subsequently, a small amount of stool was extruded through the cecum by slow and gentle squeezing. The cecum was then carefully returned to the abdominal cavity, and the incision was closed with $4 / 0$ suture lines with double layer suturing. The mice in the normal group underwent the same procedure except for ligation and puncture of the cecum. The mice were injected subcutaneously with normal saline at $30 \mathrm{~mL} / \mathrm{kg}$ after the operation and then kept in a cage. Three mice each were randomly selected in the normal group and CLP group and euthanized by neck dislocation after $12 \mathrm{~h}$ to obtain blood. The left lungs were preserved to determine the wet/dry (W/D) weight of the lung. The right lungs were weighed and stored at $-70^{\circ} \mathrm{C}$.

\section{Survival Rate Observation}

Ten mice each in the normal and CLP group were selected to observe the changes in survival rate at $0,12,24,36,48,60$, and $72 \mathrm{~h}$ after the operation. Animal death was judged by the loss of its righting reflex. The animals that were still alive after $72 \mathrm{~h}$ were statistically analyzed as having a survival time of $72 \mathrm{~h}$, and then further experiments were carried out. 


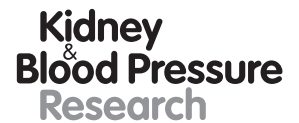

\begin{tabular}{l|l}
\hline Kidney Blood Press Res 2019;44:565-582 \\
\hline DOI: 10.1159/000500484 & $\begin{array}{l}\text { @ 2019 The Author(s). Published by S. Karger AG, Basel } \\
\text { www.karger.com/kbr }\end{array}$ \\
\hline
\end{tabular}

Ling et al.: Role of miR-203 in Septic Shock

\section{Animal Grouping and Sample Collection}

The mice were divided into the normal group (normal mice), blank group (CLP mice without transfection), negative control group (NC, CLP mice injected with blank plasmid through the caudal vein, $0.5 \mathrm{~mL} / 10 \mathrm{~g}$ ), miR-203 mimic group (CLP mice injected with 0.4 $\mathrm{pmol} / \mu \mathrm{L}$ miR-203 mimic through the caudal vein, $0.5 \mathrm{~mL} / 10 \mathrm{~g}$ ), miR-203 inhibitor group (CLP mice injected with $0.4 \mathrm{pmol} / \mu \mathrm{L}$ miR-203 inhibitor through the caudal vein, $0.5 \mathrm{~mL} / 10$ g), siRNA-VNN1 group (CLP mice injected with $0.4 \mathrm{~mol} / \mu \mathrm{L}$ siRNA-VNN1 through the caudal vein, $0.5 \mathrm{~mL} / 10 \mathrm{~g}$ ), and miR-203 mimic + siRNA-VNN1 group (CLP mice injected with 0.4 $\mathrm{mol} / \mu \mathrm{L}$ miR-203 mimic and siRNA-VNN1 plasmid through the caudal vein, $0.5 \mathrm{~mL} / 10 \mathrm{~g}$ ) (6 mice in each group; plasmids were purchased from Merck Serono, Geneva, Switzerland). After transfection, the mice were raised for $24 \mathrm{~h}$, and then one mouse was picked in each group and injected with phenobarbital into the abdominal cavity at $50 \mathrm{mg} / \mathrm{kg}$. The pulmonary artery, common carotid artery, and tracheal incubation were connected with the 4-channel data acquisition and analysis system (MP36; BIOPAC, Beijing, China) after anesthesia. The mean pulmonary artery pressure (mPAP), mean arterial pressure (MAP), airway pressure (AP), and heart rate (HR) were recorded, and blood gas analysis was carried out to measure the arterial partial pressure of oxygen $\left(\mathrm{PAO}_{2}\right)$. The experiment was repeated 3 times. After each experiment, percutaneous heart puncture was performed, and the blood was extracted from the heart. The mice were then euthanized by spinal cord dislocation, and the lung tissues were quickly extracted under aseptic condition on an ultraclean bench.

\section{Measurement of the W/D Ratio of the Lung}

After the mice were euthanized by spinal cord dislocation, the lungs were exposed and carefully isolated. The main bronchus was separated from the annular cartilage, the trachea was cut off, connective tissue was removed, and blood stains were removed. Subsequently, the lung tissues were quickly weighed on an analytical balance and placed in an incubator at $70^{\circ} \mathrm{C}$ for $24 \mathrm{~h}$. The $\mathrm{W} / \mathrm{D}$ ratio of the lungs was calculated.

\section{Hematoxylin-Eosin Staining}

The specimens were fixed with 10\% neutral formaldehyde solution for more than $24 \mathrm{~h}$. The lung tissues were collected from each group for conventional hematoxylin-eosin (HE) staining. The histopathological changes in the lung tissues were observed using an optical microscope (DMM-300D; Shanghai CaiKon Optical Instrument Co., Ltd., Shanghai, China). The HE staining results were evaluated by pulmonary pathologists. Lung injury was scored in accordance with the alveolar congestion, hemorrhage, neutrophil infiltration into the airspace or vessel wall, and thickness of the alveolar wall/hyaline membrane formation [20].

\section{Immunohistochemistry}

The lung tissues of each group were fixed with formaldehyde. Paraffin-embedded specimens were cut into 4- $\mu$ m sections, dewaxed with routine xylene, and dehydrated. After being repaired by antigen under high pressure, the sections were blocked with $10 \%$ goat serum blocking solution (CW Bio Co., Ltd., Beijing, China) at room temperature for 20 min. After excess liquid was removed, the sections were incubated overnight at $4{ }^{\circ} \mathrm{C}$ with a monoclonal rabbit anti-mouse antibody against VNN1 (1:100-1:500, ab205912; Abcam, Cambridge, MA, USA). The sections were washed three times with PBS (3 min each), incubated with biotinylated goat anti-rabbit IgG secondary antibody (1:1,000, ab6789; Abcam), and stained with $3 \mathrm{~mL}$ of diaminobenzidine (DA1010; Beijing Solabio Life Sciences Co., Ltd., Beijing, China). Five high-power fields of vision were randomly selected from each section to determine the positive staining area, percentage of positive staining to total area, and average integral optical density (OD) or mean intensity. 


\section{Kidney \\ Blood Pressure \\ Research}

\begin{tabular}{l|l}
\hline Kidney Blood Press Res 2019;44:565-582 \\
\hline DOI: 10.1159/000500484 & $\begin{array}{l}\text { ○ 2019 The Author(s). Published by S. Karger AG, Basel } \\
\text { www.karger.com/kbr }\end{array}$ \\
\hline
\end{tabular}

Ling et al.: Role of miR-203 in Septic Shock

\section{TdT-Mediated dUTP-Biotin Nick End-Labeling Assay}

Lung tissue sections from each group of mice were subjected to the TdT-mediated dUTPbiotin nick end-labeling (TUNEL) assay in accordance with the TUNEL detection kit (Roche, Basel, Switzerland). The staining results were observed under a high-power microscope. Five visual fields were observed in each group. The apoptotic rate in each visual field $(100 \%)=$ (the total number of apoptotic cells/total cells) $\times 100 \%$ and the average value were calculated.

\section{Enzyme-Linked Immunosorbent Assay}

Serum was collected from the intracardiac blood from each group of mice. The contents of tumor necrosis factor alpha (TNF- $\alpha$ ), IL-1 $\beta$, interferon gamma (IFN- $\gamma$ ), IL-10, and IL- 6 in serum were tested using an enzyme-linked immunosorbent assay (ELISA) kit. The experimental procedures were carried out based on the instructions provided by the kit (EL0001; InnoReagents, Huzhou, Zhejiang, China). The OD value was measured at $450 \mathrm{~nm}$.

\section{Dual-Luciferase Reporter Gene Assay}

According to the online prediction website microRNA.org, the target gene of miR-203 was predicted. Whether VNN1 was a direct target of miR-203 was identified by the dualluciferase reporter gene assay. Endonuclease sites (SpeI and HindIII) were introduced into the pMIR-reporter to design complementary mutation (MUT) sites of seed sequences in wild-type (WT) VNN1. The target fragment was inserted into the pMIR-reporter reporter plasmid by T4 DNA ligase after restriction endonuclease detachment. The correctly sequenced luciferase reporter plasmid of WT and MUT was cotransfected into HEK-293T cells (CRL1415; Shanghai Xinyu Biotechnology Co., Ltd., Shanghai, China) with miR-203. After 48 h of transfection, the cells were collected, lysed, and centrifuged for 3-5 min, and the supernatant was extracted. A luciferase detection kit (RG005; Beyotime Biotechnology Co., Shanghai, China) was employed to dissolve the Renilla luciferase agent in firefly luciferase binding buffer. Renilla luciferase agent was prepared with buffer solution (100 $\mu \mathrm{L} / \mathrm{sample})$ and substrate at a ratio of 1:100. A fluorescence measurement instrument was employed. Each group of samples $(20-100 \mu \mathrm{L})$ was assessed following the addition of $100 \mu \mathrm{L}$ of firefly luciferase and triturated evenly with a pipet using the cell lysate of the reporter gene as the blank control. Samples were supplemented with $100 \mu \mathrm{L}$ of Renilla luciferase agent and triturated evenly with a pipet to determine the relative light units of Renilla luciferase as the internal control. The luciferase activity was determined as the ratio of firefly to Renilla luciferase activity [21].

\section{Reverse Transcription Quantitative Polymerase Chain Reaction}

An ultrapure RNA extraction kit (D203-01; GenStar BioSolutions, Beijing, China) was used to extract total RNA. The primers were designed and synthesized by TaKaRa company (Takara Holdings Inc., Kyoto, Japan) (Table 2). RNA template, Primer Mix, dNTP Mix, DTT, RT buffer, HiFi-MMLV, and RNA-free water were dissolved on ice. The reverse transcription system consisted of a volume of $20 \mu \mathrm{L}$. The reaction solution was used for fluorescence quantitative PCR according to the instructions of the SYBR ${ }^{\circledR}$ Premix Ex Taq ${ }^{\mathrm{TM}}$ II kit (RR820A; Action-award Biotechnology Co., Ltd., Guangzhou, Guangdong, China). The PCR was performed using an ABI PRISM ${ }^{\circledR} 7300$ (Prism ${ }^{\circledR} 7300$; Shanghai Kunke instrument equipment Co., Ltd., Shanghai, China). The $2^{-\Delta \Delta \mathrm{Ct}}$ method was used to express the expression relationships of multiple genes between the experimental group and control group. Ct represented the number of amplification cycles when the real-time fluorescence intensity had reached the threshold cycle. The expression of miR-203, VNN1, AKT, caspase-3, Bcl-2, and Bax in the tissues was calculated. 
Ling et al.: Role of miR-203 in Septic Shock

Table 2. The primer sequences of miR-203, VNN1, AKT, caspase-3, Bax, Bcl-2, and GAPDH used for RT-qPCR

\begin{tabular}{ll}
\hline Gene & Primer sequence $\left(5^{\prime}\right.$-3' $\left.^{\prime}\right)$ \\
\hline miR-203 & Forward: ACACTCCAGCTGGCGTGAAATGTTTAGGACCA \\
& Reverse: CTCAACTGGTGTCGTCGTGGAGTCGGCAATTCAGTTGAGCTAGTGGT \\
\hline VNN1 & Forward: AACTGGATACCCTGTGATAACCC \\
& Reverse: GTCTCCCATGTTCGCCACAA \\
\hline AKT & Forward: CCCTGCTCCTAGTCCACCA \\
& Reverse: TGTCTCTGTTTCAGTGGGCTC \\
\hline Caspase-3 & Forward: GAATGACTGGGAGTGGGGTAGG \\
& Reverse: GCCACCTCTCCTTTCCTTACGC' \\
\hline Bax & Forward: TCGTCCATCGAGGATGACTTC \\
& Reverse: AACACCACAATTAAGGCAGGG' \\
\hline Bcl-2 & Forward: TCTCCCAGCGTGCGCCAT \\
& Reverse: TACCGCGTGCGACCCTCT \\
\hline GAPDH & Forward: AGGTCGGTGTGAACGGATTTG- \\
& Reverse: GGGGTCGTTGATGGCAACA \\
\hline
\end{tabular}

RT-qPCR, reverse transcription quantitative polymerase chain reaction; miR-203, microRNA-203; VNN1, vanin 1; Bax, BCL2-associated X protein; AKT, serine/threonine kinase 1; Bcl-2, B-cell leukemia/lymphoma; GAPDH, glyceraldehyde-3-phosphate dehydrogenase.

\section{Western Blot Analysis}

The lung tissues of mice in each group were frozen with liquid nitrogen and then ground into uniform fine powder and subsequently homogenized in an ice bath. Protein lysis buffer was added to the tissues and lysed for $30 \mathrm{~min}$ at $4^{\circ} \mathrm{C}$ with shaking every $10 \mathrm{~min}$. The sample was centrifuged at $25,764 \mathrm{~g}$ for $20 \mathrm{~min}$ at $4^{\circ} \mathrm{C}$. After the lipid layer was discarded, the supernatant was selected for detection of the protein concentration of each sample using the bicinchoninic acid (BCA) kit (20201ES76; Yeasen Biotechnology Co., Ltd., Shanghai, China). Deionized water was used to adjust the amount of protein to $30 \mu \mathrm{g} / \mathrm{lane}$. After $10 \%$ SDSpolyacrylamide gel electrophoresis, the samples were transferred onto a nitrocellulose membrane and blocked with $5 \%$ skim milk powder at $4{ }^{\circ} \mathrm{C}$ overnight. Diluted primary antibody, rabbit antibodies against VNN1 (1:1,000, ab233105), AKT (1:500, ab8805), p-AKT (ab38449, 1:1,000), caspase-3 (1:500, ab13847), Bax (1:2,000, ab32503), Bcl-2 (1:2,000, ab182858), and GAPDH (1:10,000, ab181602) were added into membrane for incubation overnight. Afterwards, PBS was used for washing membrane 3 times at room temperature, 5 min each time. The horseradish peroxidase-labeled goat anti-rabbit IgG secondary antibody (1:1,000; Wuhan Boster Biological Technology Co., Ltd., Wuhan, Hubei, China) was added into membrane for incubation at $37^{\circ} \mathrm{C}$ for $1 \mathrm{~h}$. The membrane was washed with PBS 3 times at room temperature, 5 min each time, and then immersed in the enhanced chemiluminescence reaction solution (Pierce, Waltham, MA, USA) at room temperature for $1 \mathrm{~min}$. After the liquid was absorbed, the membrane was covered with fresh-keeping film, exposed in dark room, and the results were observed after the development and fixing. GAPDH was used as an internal reference, and the ratio of the gray value of the target band to the internal reference band was taken as the relative protein expression. All antibodies were purchased from Abcam. 


\section{Kidney \\ Blood Pressure \\ Research}

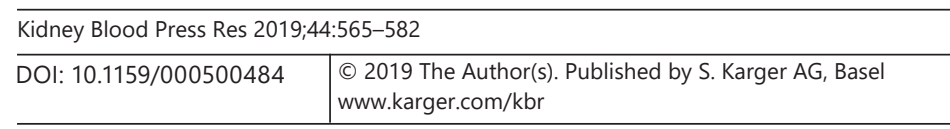

Ling et al.: Role of miR-203 in Septic Shock

Determination of Polymorphonuclear Neutrophils and Pulmonary Alveolar Macrophages in Bronchoalveolar Lavage Fluid

After surgery, the left lung of the mouse was immediately removed, and the lungs and bronchi of the mice were washed 5 times with $40 \mathrm{~mL}$ of $0.9 \%$ sodium chloride solution at $0-4{ }^{\circ} \mathrm{C}$, followed by bronchoalveolar lavage fluid (BALF) collection. The numbers of pulmonary alveolar macrophages (PAMs) and polymorphonuclear neutrophils (PMNs) in the BALF were then determined by the Rayleigh's staining solution counting method, and the number of phagocytes was simultaneously assessed by Gram staining. The percentage of phagocytes was calculated. The absolute number of cells can be determined by multiplying the number of phagocytes by the total number of cells. The BALF was centrifuged at $402 g$ for 5 min with the supernatant subsequently collected, and the protein content in the BALF was counted according to the Coomassie brilliant blue method.

Determination of Superoxide Dismutase, Malondialdehyde, Alanine Aminotransferase, and Aspartate Aminotransferase Levels

Lung tissues $(100 \mathrm{mg}$ ) from the mice in all groups were extracted, washed with saline at $4{ }^{\circ} \mathrm{C}$, and dried with a piece of filter paper after removal of the blood. Then, the tissues were homogenized with a homogenizer in an ice bath and centrifuged for $15 \mathrm{~min}$ at $10,735 \mathrm{~g}$ with a refrigerated centrifuge. An appropriate amount of supernatant was stored in an EP tube at $-20^{\circ} \mathrm{C}$. The activities of superoxide dismutase (SOD) and malondialdehyde (MDA) were measured according to the instructions of the detection kits ml001998 and ml531021 (Shanghai Enzyme-Linked Biotechnology Co., Ltd., Shanghai, China). The activity of SOD was determined by the xanthine oxidase method. The MDA levels were determined indirectly by evaluation of the OD value of the red product produced by its reaction with thiobarbituric sodium. The aminotransferase (ALT) and aspartate aminotransferase (AST) levels in serum were measured by an automatic biochemical analyzer (Olympus 2700; Olympus Optical Co., Ltd., Tokyo, Japan).

\section{Statistical Analysis}

Statistical analyses were conducted using SPSS 21.0 (IBM Corp., Armonk, NY, USA). Measurement data are expressed as the mean \pm standard deviation. The differences between the two groups were compared by the $t$ test, and multiple groups were compared by one-way analysis of variance. $p<0.05$ indicated that the difference was statistically significant.

\section{Results}

\section{A Mouse Model of Septic Shock Is Successfully Established}

Mice in the CLP group presented gradually reduced activities after $0.5 \mathrm{~h}$, trembled with their eyes closed, and produced mucous stool; they began to die after $12 \mathrm{~h}$. Only one of them

Fig. 1. GEO data analysis displays that VNN1 and miR-203 are associated with septic shock. a DEGs were in intersection of the top 80 DEGs of GSE95233, GSE57065, GSE8121, GSE4607, GSE26440, and GSE26378. b The interaction network between DEGs and disease-related genes in septic shock; the green diamond represents DEGs, and pink circle represents disease-related genes. c-e The heat maps of the top 80 DEGs in GSE95233, GSE57065, and GSE8121he horizontal coordinates represent the sample number, the vertical coordinates represent the DEGs. The upper right histogram is the color order, and each rectangle corresponds to a sample expression. f-h Expression of VNN1 in GSE4607, GSE26440, and GSE26378. i Intersection target miRNA predictions of TargetScan and microRNA for VNN1. $\mathbf{j}$ The predicted binding site of miR-203 on 3'UTR of VNN1. k Luciferase activity detection. GEO, gene expression omnibus; VNN1, vanin 1; miR-203, microRNA-203; DEGs, differentially expressed genes.

(For figure see next page.) 
Kidney

Blood Pressure

Research
Kidney Blood Press Res 2019;44:565-582
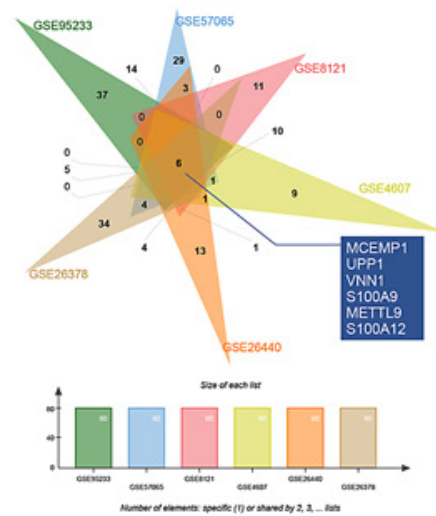

a

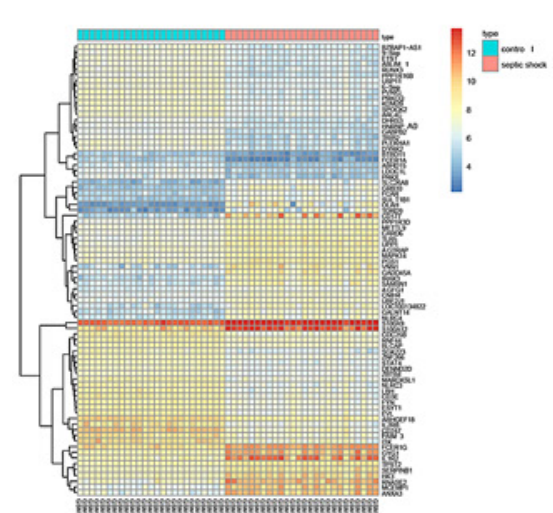

d

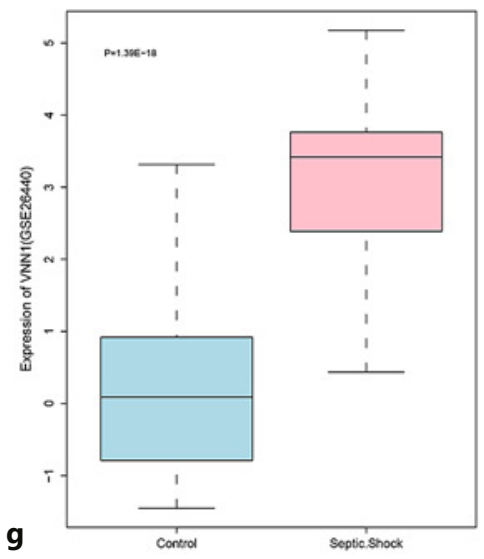

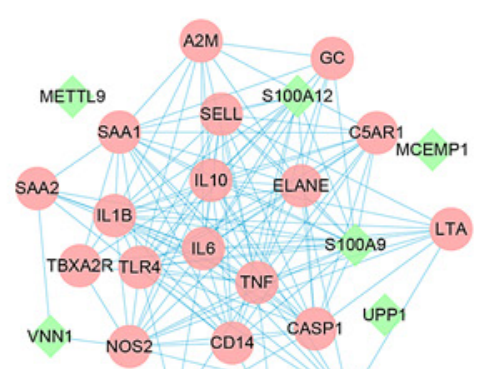

b AQP1 SLC5A1 ADM
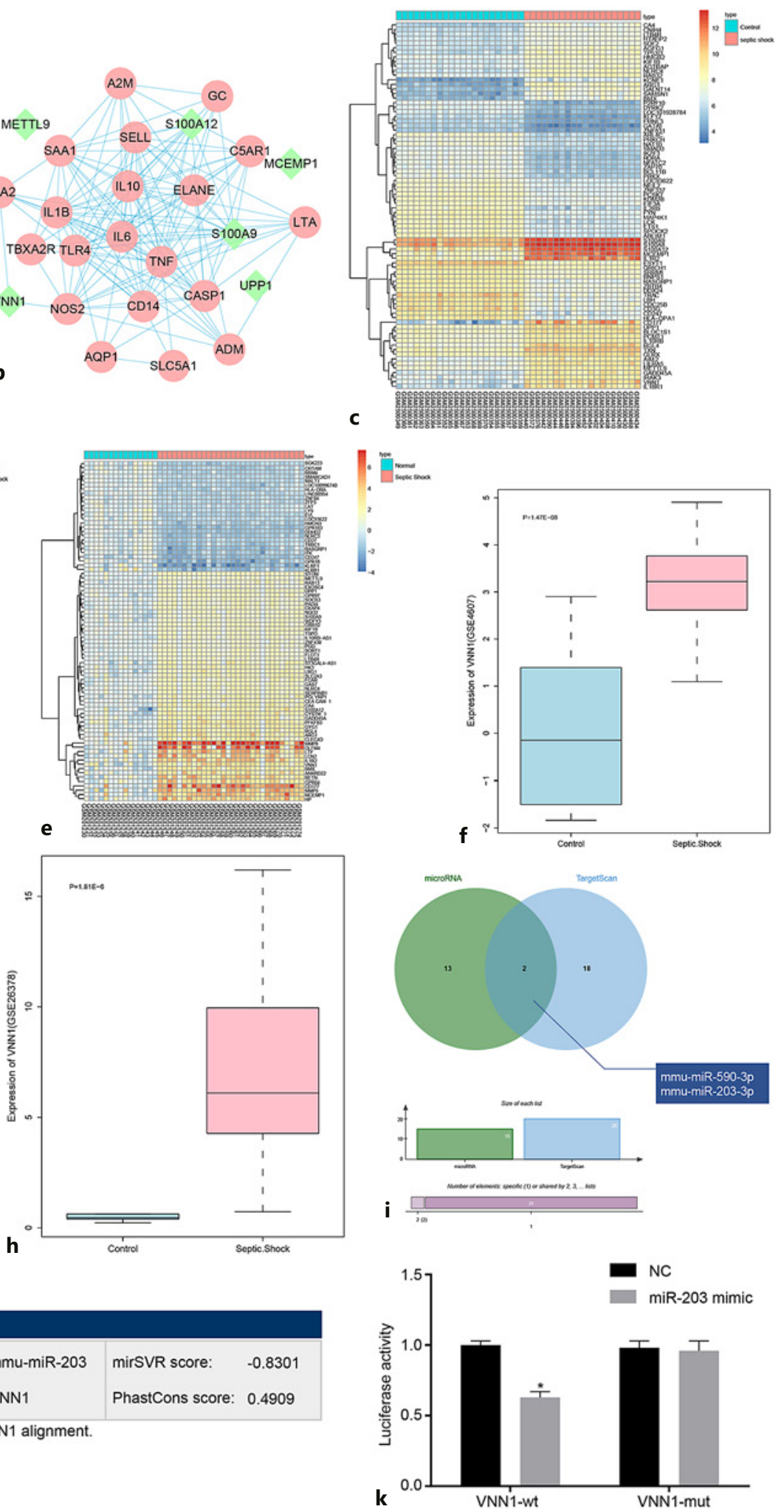

\section{C hsa-miara-379/EIF4G2 Alignment}

3' gauCACCAGGAUU - UGUAAAGUg 5' mmu-miR-203 166:5' uauGUACACCAGAUACAUUUCAg 3 ' VNN1

mirSVR score: $\quad-0.8301$ PhastCons score: 0.4909

j Mouseover miRNA mature name to see the miRNAVNN1 alignment. 
Table 3. miR-203 upregulation increases the levels of mPAP, MAP, AP, HR, and $\mathrm{PAO}_{2}$

\begin{tabular}{llllccc}
\hline Group & $n$ & $\mathrm{mPAP}, \mathrm{kPa}$ & $\mathrm{MAP}, \mathrm{mm} \mathrm{Hg}$ & $\mathrm{AP}, \mathrm{mm} \mathrm{Hg}$ & $\mathrm{HR}, \mathrm{time} / \mathrm{min}$ & $\mathrm{PAO}, \mathrm{kPa}$ \\
\hline Normal & 6 & $3.03 \pm 0.21$ & $96.51 \pm 2.21$ & $13.36 \pm 0.98$ & $435.83 \pm 12.04$ & $19.52 \pm 1.87$ \\
Blank & 6 & $1.93 \pm 0.10^{*}$ & $61.62 \pm 1.85^{*}$ & $9.57 \pm 0.45^{*}$ & $325.00 \pm 9.94^{*}$ & $11.65 \pm 0.10^{*}$ \\
NC & 6 & $1.92 \pm 0.11^{*}$ & $62.11 \pm 1.46^{*}$ & $9.63 \pm 0.42^{*}$ & $326.83 \pm 13.00^{*}$ & $11.52 \pm 0.25^{*}$ \\
miR-203 mimic & 6 & $2.21 \pm 0.13^{*, \#}$ & $74.26 \pm 1.24^{*, \#}$ & $10.81 \pm 0.51^{*, \#}$ & $359.17 \pm 16.04^{*, \#}$ & $14.15 \pm 1.31^{*, \#}$ \\
miR-203 inhibitor & 6 & $1.61 \pm 0.08^{*, \#}$ & $50.17 \pm 1.05^{*, \#}$ & $8.24 \pm 0.38^{*, \#}$ & $291.17 \pm 9.24^{*, \#}$ & $8.78 \pm 0.83^{*, \#}$ \\
siRNA-VNN1 & 6 & $2.26 \pm 0.07^{*, \#}$ & $74.45 \pm 2.71^{*, \#}$ & $10.90 \pm 0.54^{*, \#}$ & $360.83 \pm 7.91^{*, \#}$ & $14.21 \pm 1.28^{*, \#}$ \\
miR-203 mimic + & & & & & \\
$\quad$ & 6 & $2.64 \pm 0.12^{*, \#, \&}$ & $87.18 \pm 1.23^{*, \#, \&}$ & $12.04 \pm 0.76^{*, \#, \&}$ & $396.00 \pm 10.94^{*, \#, \&}$ & $16.85 \pm 1.61^{*, \#, \&}$ \\
\hline
\end{tabular}

mPAP, mean pulmonary artery pressure; MAP, mean arterial pressure; AP, airway pressure; $\mathrm{HR}$, heart rate; $\mathrm{PAO}_{2}$, arterial partial pressure of oxygen; miR-203, microRNA-203; VNN1, vanin $1 .{ }^{*} p<0.05$ vs. normal group; ${ }^{*} p<0.05$ vs. blank group and NC group; $\& p<0.05$ vs. miR-203 mimic group.

survived after $72 \mathrm{~h}$, but no mice died in the normal group. After $72 \mathrm{~h}$, the survival rate in the CLP group was $10 \%$, and the survival rate in the normal group was $100 \%$. Compared with the normal group, the CLP group had significantly increased mortality $(p<0.05)$, and the model was successfully established.

VNN1 and miR-203 Are Related to Septic Shock, and miR-203 Targets VNN1

Based on the expression profiles of GSE95233, GSE57065, GSE8121, GSE4607, GSE26440, and GSE26378, the DEGs were screened with $|\log 2 \mathrm{FC}|>2.0$ and $p<0.05$, and the top 80 DEGs were compared in each dataset. Six genes in the intersection (MCEMP1, UPP1, VNN1, S100A9, METTL9, and S100A12) were identified as candidate genes by the Venn diagram (Fig. 1a). The DisGeNET database was used to search for septic shock-related genes, and the top 20 genes were selected as disease-related genes. The disease-related gene and DEG interaction network was constructed (Fig. 1b), in which VNN1, S100A9, and S100A12 were the three DEGs that interacted with the disease-related gene. However, S100A9 [22] and S100A12 [23] have been reported to be associated with septic shock; therefore, the aberrantly expressed VNN1 drew our attention. Figure 1c-e shows the expression profiles of the top 80 DEGs in GSE95233, GSE7065, and GSE8121, respectively, and VNN1 expression was higher in patients with septic shock in these three chips. In addition, the VNN1 expression in the GSE4607 (Fig. 1f), GSE26440 (Fig. 1g), and GSE26378 (Fig. 1h) chips was analyzed, and VNN1 showed abnormally high expression in septic shock. There were two miRNAs in intersection (mmu-miR-590-3p and mmu-miR-203-3p) in the top 20 miRNAs of the two databases, suggesting that these two miRNAs might regulate VNN1 (Fig. 1i). The Context++ score is a predicted value of the miRNA-mRNA relationship in TargetScan [24]. The Context++ scores of mmu-miR-590-3p and mmu-miR-203-3p were -0.02 and -0.23 , respectively, and therefore we focused on whether miR-203 regulated VNN1 expression during septic shock. According to the online prediction website analysis, there was a binding site between miR-203 and the VNN13'-untranslated region (3'UTR). VNN1 was the target gene of miR-203 (Fig. 1j). A dual-luciferase reporter gene assay was used to verify that VNN1 was the target of miR-203 (Fig. 1k). Compared with the NC group, the miR-203 mimic significantly inhibited the activity of luciferase in the VNN1 WT 3'UTR $(p<0.05)$, while miR-203 had no significant effect on the activity of luciferase in MUT 3'UTR $(p>0.05)$. miR-203 could specifically bind to VNN1-3'-UTR and downregulate the expression of the VNN1 gene at the posttranscriptional level. 
Fig. 2. Upregulated microRNA-203 (miR-203) reduces the wet/dry ratio of lung $(n=6) . * p<$ 0.05 vs. normal group; ${ }^{\#} p<0.05$ vs. blank group and negative control (NC) group; \& $p<0.05$ vs. miR203 mimic group.

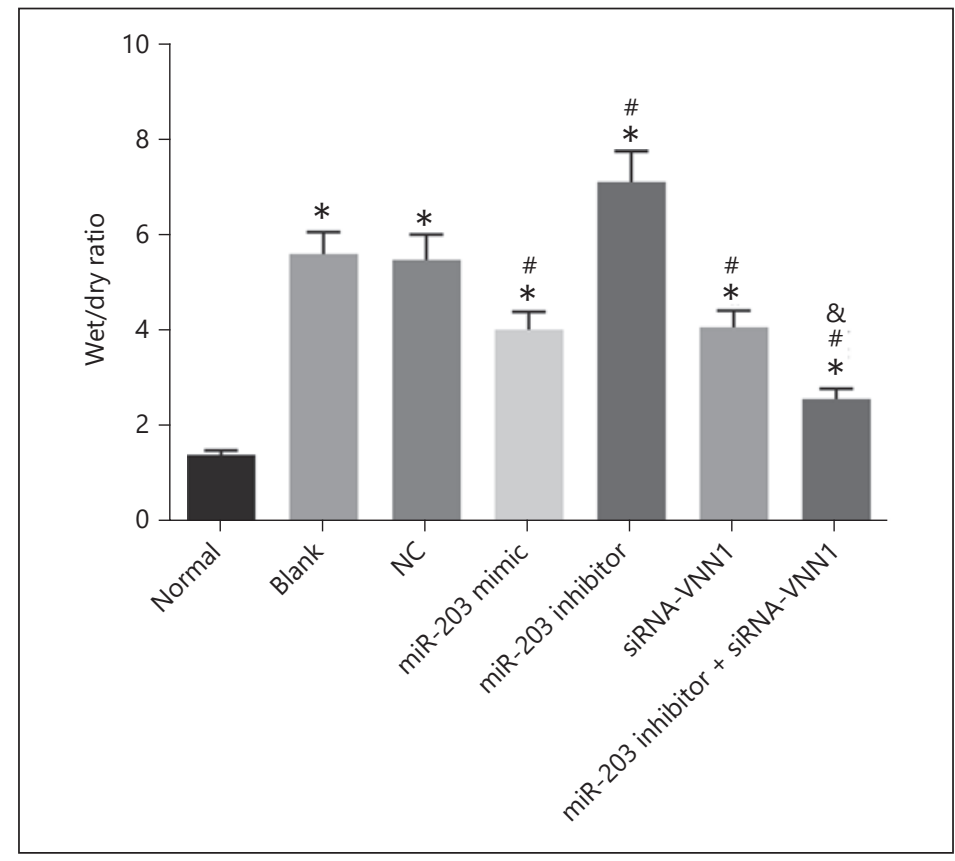

Upregulated miR-203 Increases the Levels of $M P A P, M A P, A P, H R$, and $P_{2} O_{2}$

The mPAP, MAP, AP, HR, and $\mathrm{PAO}_{2}$ levels were significantly lower in the blank, NC, miR-203 mimic, miR-203 inhibitor, siRNA-VNN1, and miR-203 mimic + siRNA-VNN1 groups than in the normal group $(p<0.05)$. Compared with the blank and NC groups, the miR-203 mimic, siRNAVNN1 and miR-203 mimic + siRNA-VNN1 groups had significantly higher mPAP, MAP, AP, HR, and $\mathrm{PAO}_{2}$ levels $(p<0.05)$, whereas the miR-203 inhibitor group showed significantly decreased mPAP, MAP, AP, HR, and $\mathrm{PAO}_{2}$ levels $(p<0.05)$. Compared with the miR-203 mimic group, the miR-203 mimic + siRNA-VNN1 group demonstrated significantly elevated mPAP, MAP, AP, HR, and $\mathrm{PAO}_{2}$ levels $(p<0.05)$ (Table 3). Collectively, these data indicate that miR-203 overexpression can alleviate lung injury by increasing the mPAP, MAP, AP, $\mathrm{HR}$, and $\mathrm{PAO}_{2}$ levels.

\section{Upregulated miR-203 Reduces the W/D Ratio of the Lung}

To further verify our findings, we examined the W/D ratio of the lung in mouse models of septic shock. Compared with the normal group, the blank, NC, miR-203 mimic, miR-203 inhibitor, siRNA-VNN1, and miR-203 mimic + siRNA-VNN1 groups displayed a significantly increased W/D ratio of lung $(p<0.05)$. Compared with the blank group, there was no significant difference in the W/D ratio of the lung in the NC group $(p>0.05)$. Compared with the blank and NC groups, the miR-203 mimic, siRNA-VNN1, and miR-203 mimic + siRNA-VNN1 groups exhibited a significantly reduced $\mathrm{W} / \mathrm{D}$ ratio $(p<0.05)$, whereas the miR-203 inhibitor group had a significantly increased W/D ratio of lung $(p<0.05)$. Compared with the miR-203 mimic group, the miR-203 mimic + siRNA-VNN1 group displayed a significantly lower W/D ratio of lung $(p<0.05)$ (Fig. 2). Taken together, our data suggest that the upregulation of miR-203 decreases the W/D ratio of lung.

VNN1 Is Highly Expressed and Induces Pathological Changes in the Lung Tissues of Mice with Septic Shock

HE staining was used to assess pathological changes and examine the severity of lung injury in mice treated with miR-203 inhibitor, mimic and/or VNN1 siRNA. HE staining showed 
Kidney

Blood Pressure

Research

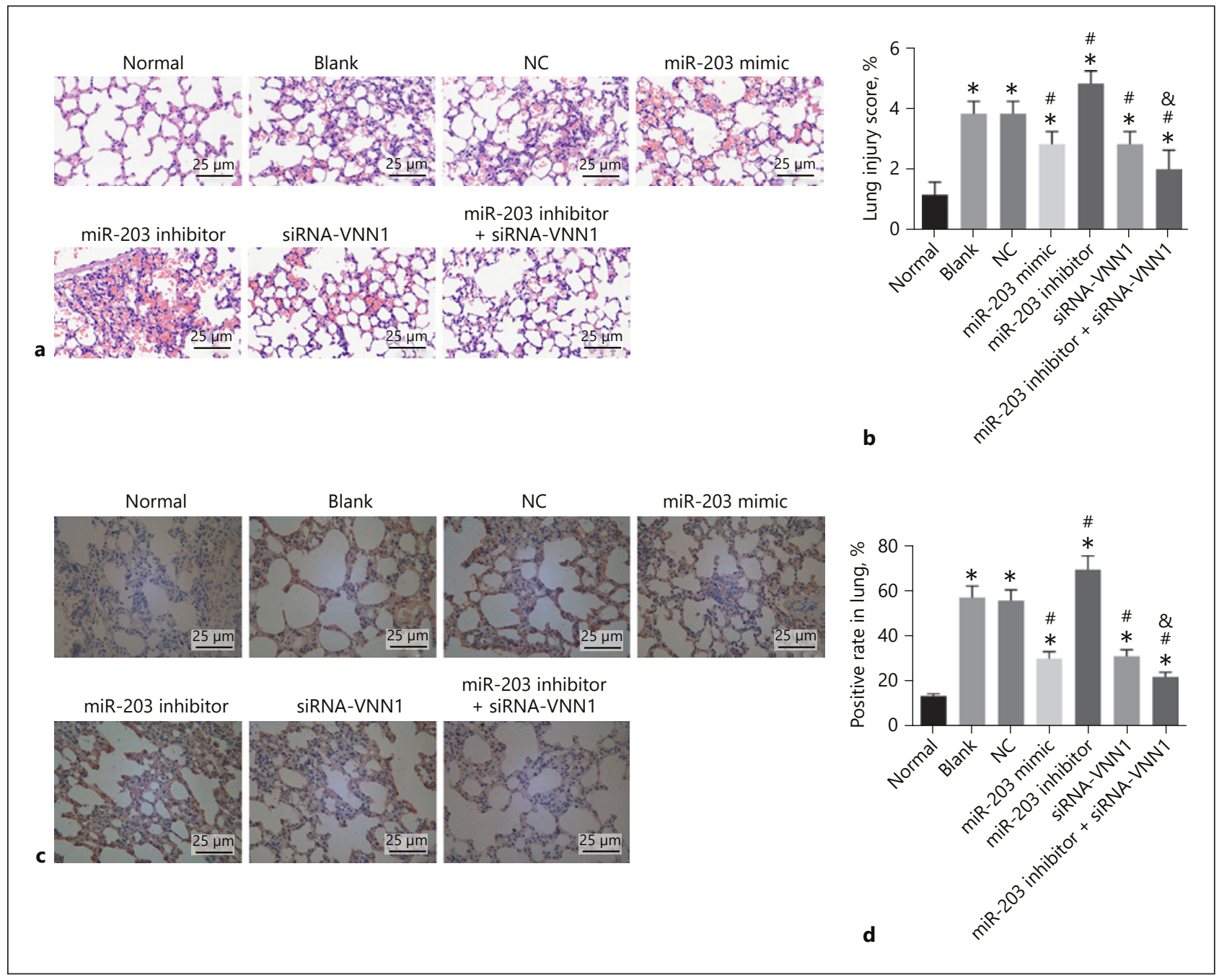

Fig. 3. Vanin-1 (VNN1) is highly expressed and involved in the histopathological changes of lung tissues of mice with septic shock. a Hematoxylin-eosin staining in lung tissues of mice in each group after transfection. $\times 400$. b Lung injury score of mice in each group. $\mathbf{c}$ Immunohistochemical staining of VNN1 in the lung tissues. $\times 400$. $\mathbf{d}$ The quantitative analysis of VNN1-positive rate in the lung tissues. ${ }^{*} p<0.05$ vs. normal group; ${ }^{\#} p<$ 0.05 vs. blank group and negative control (NC) group; ${ }^{\&} p<0.05$ vs. microRNA-203 (miR-203) mimic group.

that the structure of the lung tissues was normal and free of inflammation in the normal group. In the blank and NC groups, lung sections of mice showed alveolar structure injury with alveolar septum thickening and destruction, inflammatory cell infiltration and a significantly elevated lung injury score. However, the pathological changes in lung tissues were attenuated, and the lung injury scores were diminished in the miR-203 mimic, siRNA-VNN1, and miR-203 mimic + siRNA-VNN1 groups. Moreover, histological analysis showed that compared with the mice in the blank group, the mice in the miR-203 inhibitor group had substantially more severe lung injury and enhanced lung injury scores (Fig. 3a, b). VNN1 expression was measured by immunohistochemistry (Fig. 3c, d). Positive expression of VNN1 was cytoplasmic staining. The positive rate of VNN1 expression in the blank and NC groups was significantly higher than in the normal group $(p<0.05)$. Compared with the blank and NC group, the miR-203 mimic, siRNA-VNN1, miR-203 mimic + siRNA-VNN1 groups had signif- 


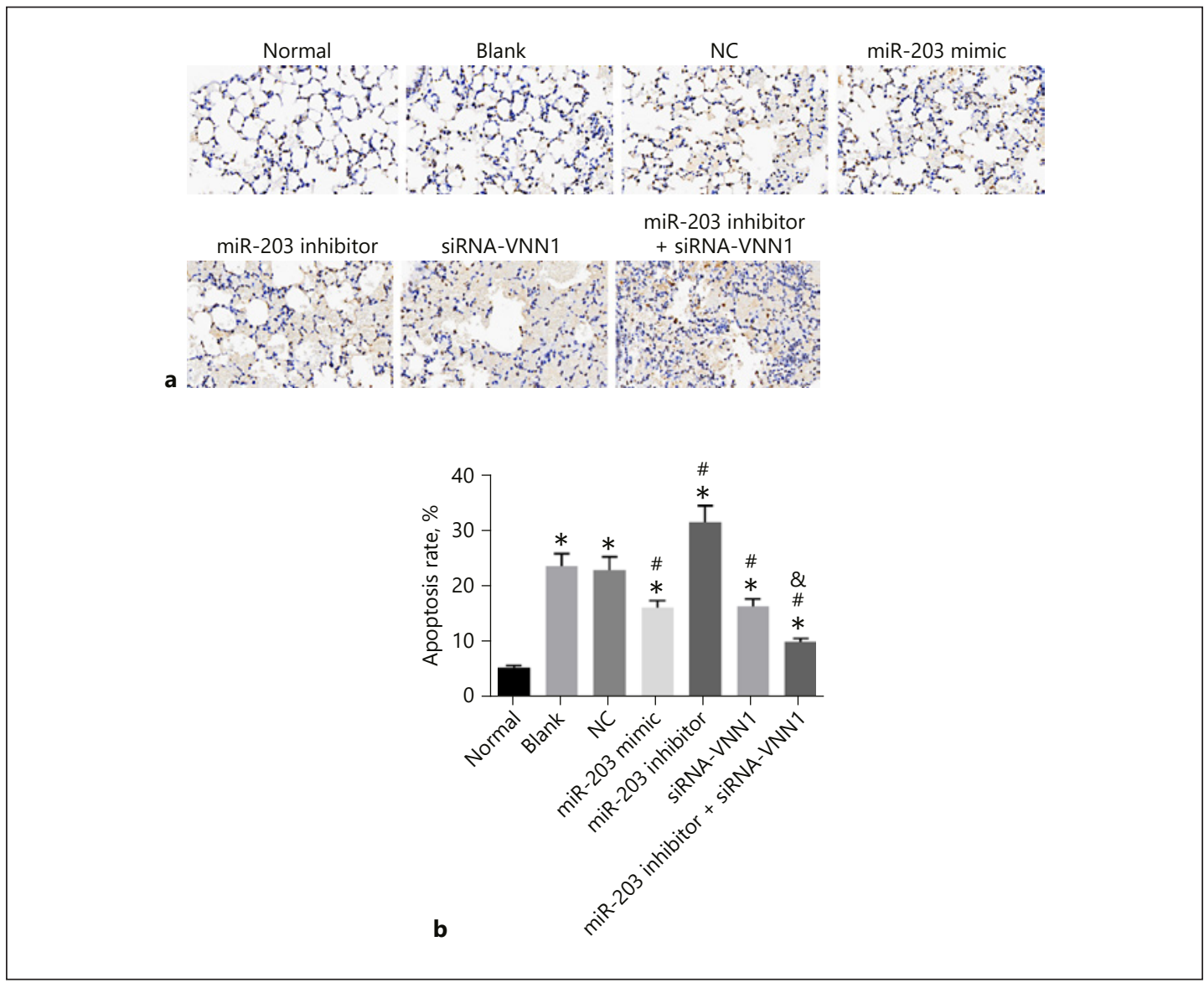

Fig. 4. Upregulated microRNA-203 (miR-203) decreases lung cell apoptosis in mice with septic shock. a TUNEL staining reflecting apoptotic lung tissues. $\mathbf{b}$ The apoptosis rate of lung tissues. ${ }^{*} p<0.05$ vs. normal group; ${ }^{\#} p<0.05$ vs. blank and negative control (NC) groups; ${ }^{\&} p<0.05$ vs. miR-203 mimic group.

icantly lower positive expression rates of VNN1 in lung tissues $(p<0.05)$; the positive expression rate of VNN1 in lung tissues in the miR-203 inhibitor group was significantly higher $(p<0.05)$. Compared with the miR-203 mimic group, the miR-203 mimic + siRNAVNN1 group had a significantly decreased positive expression rate of VNN1 in lung tissues $(p<0.05)$. The results suggest that VNN1 was enriched in lung tissues of mice with septic shock, and knockdown of VNN1 expression might accentuate lung tissue injury in these mice.

\section{Upregulated miR-203 Attenuates Apoptosis in Lung Tissues via the Decline of VNN1}

To investigate whether miR-203 could reduce lung cell apoptosis and thus protect the lungs, we next measured the level of apoptosis in the lung tissues using a TUNEL assay (Fig. 4). The apoptotic cells showed yellow staining of the nucleus. Compared with the normal group, the other groups displayed a significantly higher apoptosis rate $(p<0.05)$. Compared with the blank and NC groups, the miR-203 mimic, siRNA-VNN1, miR-203 mimic + siRNA-VNN1 groups had a significantly decreased apoptosis rate $(p<0.05)$, while the apoptosis rate in the miR-203 inhibitor group was significantly elevated $(p<0.05)$. Compared with the miR-203 mimic group, the miR-203 mimic + siRNA-VNN1 group demonstrated a significantly lower apoptosis rate $(p<0.05)$. These results indicate that miR-203 overexpression and VNN1 silencing contribute to the reduction of lung cell apoptosis. 
Table 4. Overexpression of miR-203 inhibits the contents of TNF- $\alpha$, IL-1 $\beta$, IFN- $\gamma$, IL-10, and IL-6

\begin{tabular}{lllllcc}
\hline Group & $n$ & TNF- $\alpha, \mathrm{pg} / \mathrm{mL}$ & $\mathrm{IL}-1 \beta, \mathrm{pg} / \mathrm{mL}$ & $\mathrm{IFN}-\gamma, \mathrm{pg} / \mathrm{mL}$ & $\mathrm{IL}-10, \mathrm{pg} / \mathrm{mL}$ & $\mathrm{IL}-6, \mathrm{pg} / \mathrm{mL}$ \\
\hline Normal & 6 & $42.37 \pm 3.24$ & $21.83 \pm 1.73$ & $16.13 \pm 1.38$ & $53.28 \pm 4.31$ & $37.95 \pm 2.86$ \\
Blank & 6 & $232.41 \pm 16.32^{*}$ & $71.83 \pm 6.35^{*}$ & $33.40 \pm 2.75^{*}$ & $133.75 \pm 11.14^{*}$ & $121.57 \pm 10.02^{*}$ \\
NC & 6 & $234.19 \pm 17.26^{*}$ & $73.61 \pm 5.98^{*}$ & $35.52 \pm 2.42^{*}$ & $136.92 \pm 12.03^{*}$ & $123.16 \pm 11.24^{*}$ \\
miR-203 mimic & 6 & $161.58 \pm 12.94^{*, \#}$ & $52.97 \pm 4.62^{*, \#}$ & $27.85 \pm 2.29^{*, \#}$ & $103.26 \pm 10.13^{*, \#}$ & $90.03 \pm 8.39^{*, \#}$ \\
miR-203 inhibitor & 6 & $305.00 \pm 23.73^{*, \#}$ & $90.05 \pm 7.32^{*, \#}$ & $41.69 \pm 3.78^{*, \#}$ & $156.52 \pm 13.70^{*, \#}$ & $148.61 \pm 13.42^{*, \#}$ \\
siRNA-VNN1 & 6 & $158.47 \pm 11.76^{*, \#}$ & $53.22 \pm 4.28^{*, \#}$ & $28.06 \pm 2.17^{*, \#}$ & $100.54 \pm 9.87^{*, \#}$ & $89.26 \pm 7.58^{*, \#}$ \\
miR-203 mimic + & & & & & \\
\multicolumn{1}{l}{ siRNA-VNN1 } & 6 & $106.31 \pm 9.28^{*, \#, \&}$ & $38.24 \pm 2.35^{*, \#, \&}$ & $21.78 \pm 1.95^{*, \#, \&}$ & $75.53 \pm 4.51^{*, \#, \&}$ & $64.71 \pm 5.03^{*, \#, \&}$ \\
\hline
\end{tabular}

miR-203, microRNA-203; VNN1, vanin 1 ; TNF- $\alpha$, tumor necrosis factor alpha; IL, interleukin; IFN- $\gamma$, interferon gamma. ${ }^{*} p<0.05$ vs. normal group; ${ }^{\#} p<0.05$ vs. blank group and NC group; ${ }^{\&} p<0.05$ vs. miR-203 mimic group.

Table 5. Overexpression of miR-203 upregulates SOD activity while it downregulates MDA, ALT, and AST contents

\begin{tabular}{lllll}
\hline Group & SOD, U/mg & MDA, $\mu \mathrm{mol} / \mathrm{mg}$ & ALT, U/L & AST, U/L \\
\hline Normal & $83.29 \pm 6.13$ & $11.10 \pm 0.94$ & $47.38 \pm 3.77$ & $135.59 \pm 19.04$ \\
Blank & $48.69 \pm 4.17^{*}$ & $24.64 \pm 2.11^{*}$ & $102.36 \pm 9.53^{*}$ & $337.41 \pm 21.45^{*}$ \\
NC & $49.36 \pm 4.08^{*}$ & $25.09 \pm 2.31^{*}$ & $103.58 \pm 10.05^{*}$ & $342.61 \pm 25.01^{*}$ \\
miR-203 mimic & $59.23 \pm 4.75^{*, \#}$ & $19.16 \pm 1.63^{*, \#}$ & $80.95 \pm 5.81^{*, \#}$ & $259.51 \pm 22.14^{*, \#}$ \\
miR-203 inhibitor & $37.55 \pm 3.28^{*, \#}$ & $28.67 \pm 2.30^{*, \#}$ & $121.95 \pm 11.62^{*, \#}$ & $476.26 \pm 35.93^{*, \#}$ \\
SiRNA-VNN1 & $61.03 \pm 5.12^{*, \#}$ & $19.34 \pm 1.41^{*, \#}$ & $81.31 \pm 6.53^{*, \#}$ & $266.51 \pm 24.34^{*, \#}$ \\
miR-203 mimic + siRNA-VNN1 & $70.49 \pm 6.13^{*, \#, \&}$ & $15.82 \pm 1.25^{*, \#, \&}$ & $66.27 \pm 4.74^{*, \#, \&}$ & $199.31 \pm 23.08^{*, \#, \&}$ \\
\hline
\end{tabular}

miR-203, microRNA-203; VNN1, vanin 1; SOD, superoxide dismutase; MDA, malondialdehyde; ALT, alanine aminotransferase; AST, aspartate aminotransferase. ${ }^{*} p<0.05$ vs. normal group; ${ }^{*} p<0.05$ vs. blank group and NC group; ${ }^{\&} p<0.05$ vs. miR-203 mimic group.

Upregulated miR-203 Inhibits the Serum Levels of TNF- $\alpha, I L-1 \beta$, IFN- $\gamma, I L-10$, and IL-6

A systemic inflammatory response caused by overexpression of inflammatory mediators is deemed as the main pathogenesis of septic shock [25]. Thus, we used ELISA to evaluate relevant inflammatory cytokines. Compared with the normal group, the blank, NC, miR-203 mimic, miR-203 inhibitor, siRNA-VNN1, and miR-203 mimic + siRNA-VNN1 groups had significantly increased contents of TNF- $\alpha$, IL-1 $\beta$, IFN- $\gamma$, IL-10, and IL- 6 in the blood $(p<0.05)$. Compared with the blank and NC groups, the miR-203 mimic, siRNA-VNN1, and miR-203 mimic + siRNA-VNN1 groups had significantly reduced contents of TNF- $\alpha$, IL-1 $\beta$, IFN- $\gamma$, IL-10, and IL-6 $(p<0.05)$, while the miR-203 inhibitor group showed significantly higher contents of TNF- $\alpha$, IL-1 $\beta$, IFN- $\gamma$, IL-10, and IL-6 ( $p<0.05)$. Compared with the miR-203 mimic group, the miR-203 mimic + siRNA-VNN1 group demonstrated significantly reduced contents of TNF- $\alpha$, IL-1 $\beta$, IFN- $\gamma$, IL-10, and IL-6 $(p<0.05)$ (Table 4). Taken together, our data suggest that miR-203 ameliorates lung injury and inflammatory responses.

Upregulated miR-203 Increases SOD Activity while Decreasing the Contents of MDA, ALT, and AST in Lung Tissues

Next, oxidative stress indexes and blood biochemical indexes were detected. Compared with the normal group, the blank, NC, miR-203 mimic, miR-203 inhibitor, siRNA-VNN1, and miR-203 mimic + siRNA-VNN1 groups had significantly decreased SOD activity and significantly increased contents of MDA, ALT, and AST ( $p<0.05)$. Compared with the blank and NC groups, the miR-203 mimic, siRNA-VNN1, and miR-203 mimic + siRNA-VNN1 groups showed significantly higher SOD activity and significantly decreased contents of MDA, ALT, and AST $(p<0.05)$, while the SOD 
Ling et al.: Role of miR-203 in Septic Shock

Table 6. miR-203 upregulates the PMN and PAM in BALF

\begin{tabular}{lll}
\hline Group & PAM number $/ \times 10^{9}$ & PMN number $/ \times 10^{9}$ \\
\hline Normal & $0.52 \pm 0.04$ & $0.18 \pm 0.02$ \\
Blank & $1.25 \pm 0.10^{*}$ & $1.38 \pm 0.12^{*}$ \\
NC & $1.27 \pm 0.12^{*}$ & $1.41 \pm 0.09^{*}$ \\
miR-203 mimic & $2.19 \pm 0.23^{*, \#}$ & $2.36 \pm 0.18^{*, \#}$ \\
miR-203 inhibitor & $0.96 \pm 0.07^{*, \#}$ & $0.73 \pm 0.06^{*, \#}$ \\
siRNA-VNN1 & $2.18 \pm 0.25^{*, \#}$ & $2.35 \pm 0.20^{*, \#}$ \\
miR-203 mimic + siRNA-VNN1 & $1.73 \pm 0.14^{*, \#, \&}$ & $1.85 \pm 0.15^{*, \#, \&}$ \\
\hline
\end{tabular}

PMN, polymorphonuclear neutrophils; PAM, pulmonary alveolar macrophage; BALF, bronchoalveolar lavage fluid. ${ }^{*} p<0.05$ vs. normal group; ${ }^{\#} p<0.05$ vs. blank and NC groups; ${ }^{\&} p<0.05$ vs. miR-203 mimic group.

activity in the miR-203 inhibitor group was significantly decreased, and the contents of MDA, ALT, and AST were significantly increased $(p<0.05)$. Compared with the miR-203 mimic group, the miR-203 mimic + siRNA-VNN1 group displayed significantly increased SOD activity as well as significantly decreased contents of MDA, ALT, and AST $(p<0.05)$ (Table 5).

Upregulated miR-203 Increases PMNs and PAMs in BALF

Subsequently, the PMNs and PAMs in BALF were determined. Compared with the normal group, the blank, NC, miR-203 mimic, miR-203 inhibitor, siRNA-VNN1, and miR-203 mimic + siRNA-VNN1 groups demonstrated significantly increased PMNs and PAMs in BALF ( $p<$ $0.05)$. There were no differences in PMNs and PAMs in BALF between the blank and NC groups $(p>0.05)$. Compared with the blank and NC groups, the miR-203 mimic, siRNA-VNN1, and miR-203 mimic + siRNA-VNN1 groups showed significantly increased PMN and PAM contents in BALF $(p<0.05)$, while those in the BALF of the miR-203 inhibitor group were significantly reduced. Compared with the miR-203 mimic group, the miR-203 mimic + siRNA-VNN1 group had significantly reduced PMN and PAM contents in BALF $(p<0.05)$ (Table 6).

\section{Upregulated miR-203 Inhibits the Expression of VNN1 by Activating the AKT Signaling}

Pathway

RT-qPCR (reverse transcription quantitative polymerase chain reaction) and Western blot analysis were applied to test the expression of miR-203, VNN1, AKT, and apoptosisrelated factors (caspase-3, Bcl-2, Bax). The results showed that compared with the normal group, the blank group had significantly increased mRNA and protein expression of VNN1, caspase-3, Atg5, and Bax, but significantly decreased expression of miR-203, AKT, p-AKT, and Bcl-2 in lung tissues $(p<0.05)$. There were no significant differences between the lung tissues of the blank and NC groups in terms of the expression of miR-203, VNN1, AKT, p-AKT, caspase-3, Bcl-2, and Bax $(p>0.05)$. Compared with the blank and NC groups, the miR-203 mimic, siRNA-VNN1, and miR-203 mimic + siRNA-VNN1 groups showed significantly decreased mRNA and protein expression of VNN1, caspase-3, and Bax, but significantly increased expression of miR-203, AKT, p-AKT, and Bcl-2 in lung cells $(p<0.05)$. In the miR-203 inhibitor group, the expression of VNN1, caspase-3, and Bax was increased, while expression of miR-203, AKT, p-AKT, and Bcl-2 was decreased in lung cells $(p<0.05)$. Compared with the miR-203 mimic group, there was no significant difference in miR-203 expression in the miR-203 mimic + siRNA-VNN1 group $(p>0.05)$, but the mRNA and protein expression of VNN1, caspase-3, and Bax was significantly decreased while expression of AKT, p-AKT, and Bcl-2 was significantly elevated $(p<0.05)$ (Fig. 5a-c). 


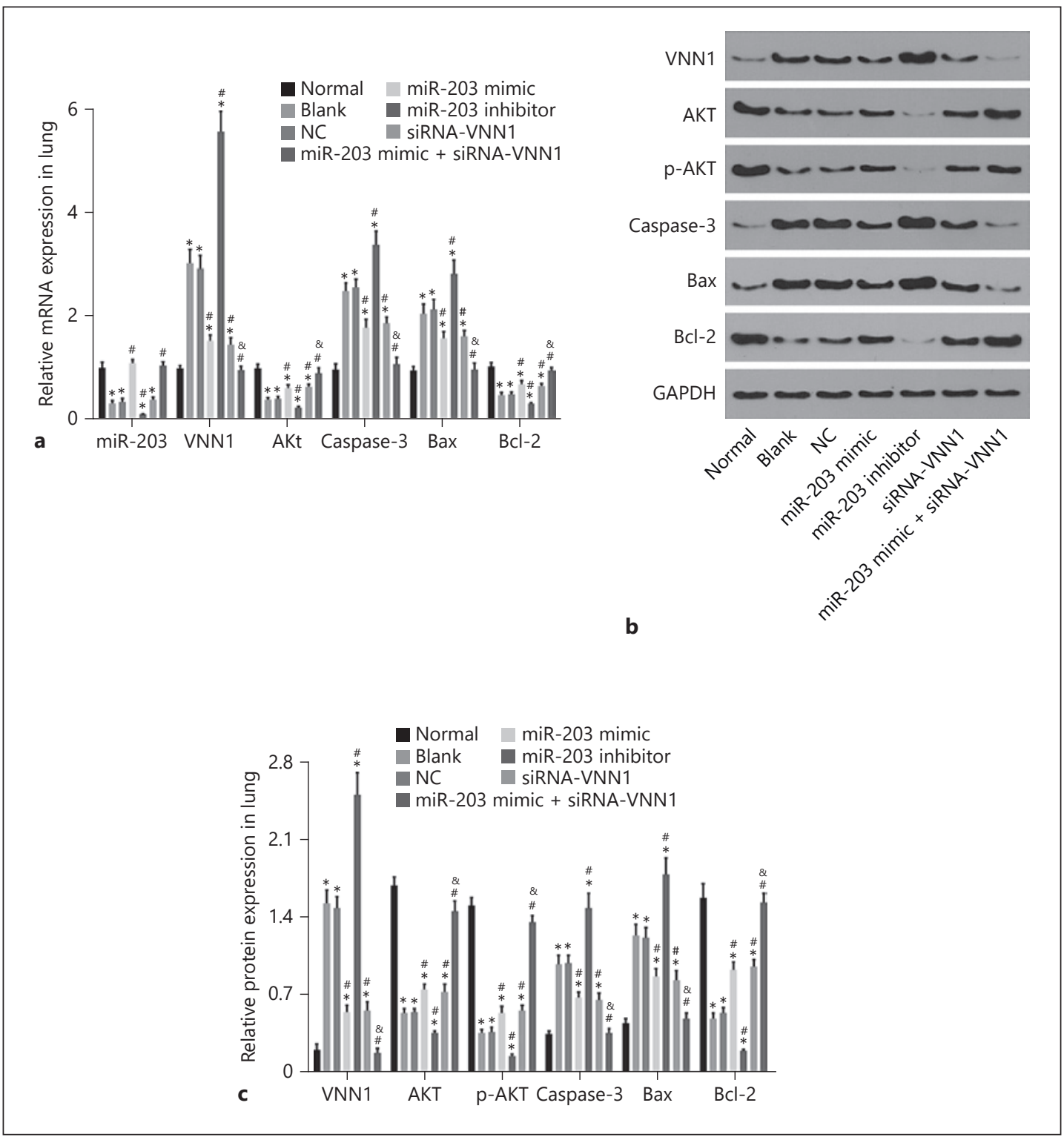

Fig. 5. Upregulated microRNA-203 (miR-203) represses vanin-1 (VNN1) expression by activating the serine/threonine kinase 1 (AKT) signaling pathway in lung tissues of mice with septic shock. a The miR-203 expression and mRNA expression of VNN1, caspase-3, BCL2-associated X protein (Bax), AKT, and B-cell leukemia/lymphoma (Bcl-2) in response to the treatment with miR-203 mimic, miR-203 inhibitor, si-VNN1, miR-203 mimic + si-VNN1 in lung tissues. b The gray value of VNN1, p-AKT, caspase-3, Bax, AKT, and Bcl-2 protein bands in lung tissues were determined by Western blot analysis. c The protein levels of VNN1, AKT, caspase-3, Bax, Bcl-2, as well as the extent of AKT phosphorylation in lung tissues were determined by Western blot analysis. ${ }^{*} p<0.05$ vs. normal group; ${ }^{\#} p<0.05$ vs. blank and negative control (NC) groups; ${ }^{\&} p<0.05$ vs. miR-203 mimic group.

\section{Discussion}

Representing an urgent problem in medicine, septic shock resulted in a greater than $80 \%$ in-hospital death rate 30 years ago, and it is still a life-threatening disease with a mortality approaching $20-30 \%$ under conditions of advanced treatment methods and better moni- 


\section{Kidney \\ Blood Pressure \\ Research}

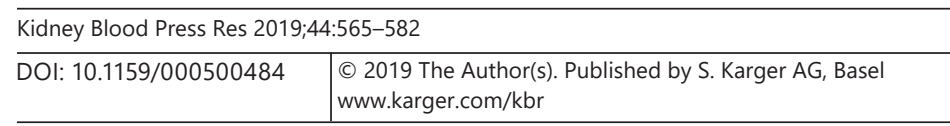

Ling et al.: Role of miR-203 in Septic Shock

Fig. 6. Molecular mechanisms involved in miR-203 binding to VNN1 and regulation of lung injury in septic shock. miR-203 is downregulated, while VNN1 is upregulated in mice with septic shock. With miR-203 overexpression, VNN1 expression was inhibited, and the apoptosis was promoted via the activation of the AKT signaling pathway.

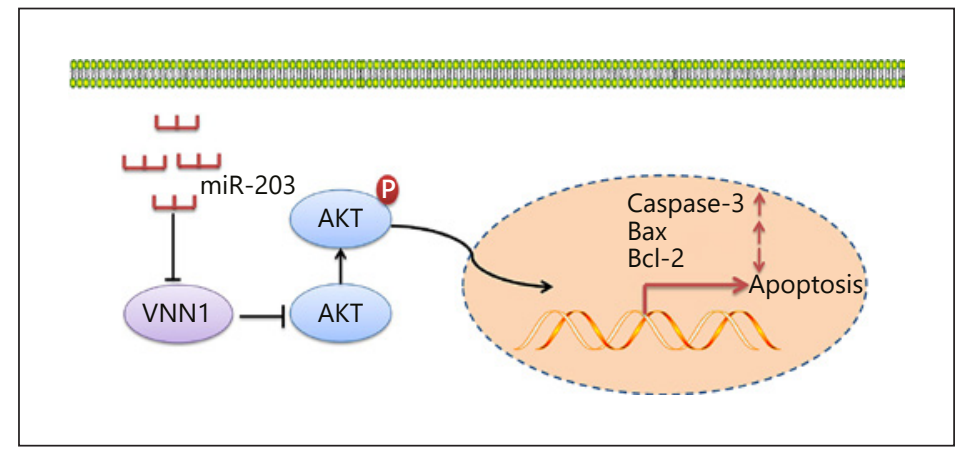

toring [26]. miRNAs have been shown to play a crucial role in cell proliferation, apoptosis, control of inflammation, lymphocyte response, and regulation of sepsis [27]. In this study, we explored the mechanism of the miR-203, VNN1, and AKT signaling pathway in septic shock. Accordingly, this study demonstrated that miR-203 negatively targeted the VNN1 gene through the AKT signaling pathway, and overexpression of miR-203 alleviated lung injury in septic shock by suppressing the inflammatory response and oxidative stress.

According to our research, miR-203 presented lower expression, while VNN1 displayed higher expression. Moreover, the present study showed that miR-203 could induce activation of the AKT signaling pathway by binding to VNN1. miR-203 has been reported to be downregulated or upregulated in different cancers, serving a role as a tumor suppressor or promoter [28]. Additionally, inactivation of VNN1 decreases the formation of tumor and vascular smooth muscle proliferation and migration [29].

One important finding of our study was that miR-203 negatively regulated the inflammatory response in lung tissues from mice with septic shock by activating the AKT signaling pathway through inhibition of VNN1. Acute lung injury features severe inflammation within the lung, leading to an impaired alveolar endothelial barrier, which contributes to an inflow of excessive neutrophils and other inflammatory cells, as well as the release of inflammatory and cytotoxic mediators [7]. A previous study has shown that at 1-3 days, the levels of IL-6, TNF- $\alpha$, IL-1 $\beta$, and IL-10 in serum are significantly increased in lung inflammation and injury [30]. Binding of IL-6, a heterotrimeric membrane-associated protein, to gp80 promotes homodimerization of gp130 and consequently activates downstream pathways, such as the AKT and JAK2/STAT3 signaling pathway [31]. Overexpression of miR-203 can downregulate the level of TNF- $\alpha$ and IL-6 to regulate lipopolysaccharide or bacille Calmette-Guérin [32]. Moreover, the overexpression of Sirt1, which is positively correlated with miR-203, has been found to attenuate LPS-induced inflammatory injury by regulating the AKT signaling pathway [33], consistent with our results showing that upregulation of miR-203 inhibited the levels of TNF- $\alpha$, IL-1 $\beta$, IFN- $\gamma$, IL-10, and IL-6, eventually reducing inflammatory responses.

The present study also showed that miR-203 worked as a suppressor of lung cell apoptosis and oxidative stress by activating the AKT signaling pathway and inhibiting VNN1, as indicated by downregulated caspase-3 and Bax, while it upregulated Bcl-2. Yu and Yao [34] reported that in septic shock, SOD activity was reduced, and MDA contents were increased. SOD, a kind of antioxidant, functions in the first line of protection against oxidative stress [35]. As the most important product of cell lipid oxidation, MDA can exacerbate the injury to the cell membrane, thus reflecting the severe extent of membrane injury [36]. Moreover, our data illustrated that SOD activity was elevated, while the contents of MDA, ALT, and AST were reduced, after miR-203 overexpression and/or VNN1 knockdown treatment in mouse models of septic shock. Oxidative stress is implicated in the expression of apoptosis-related proteins 
[37]. A recent study has shown that differential regulation of miR-203 can promote cell death and is also involved in cell apoptosis and autophagy in breast cancer [38]. The growth of lung cancer can be inhibited both in vitro and in vivo by the promotion of apoptosis-related signaling pathways, such as the AKT signaling pathway, and the suppression of proliferation [39].

In summary, our preliminary findings suggest that miR-203 targeting VNN1 may alleviate lung injury in mice with septic shock through activating the AKT signaling pathway (Fig. 6), providing a novel idea and new strategy for the treatment of septic shock. In the future, we will extend the time period of our study to gain more subjects to explore the effects of miR-203 on lung injury in patients with septic shock to further confirm our conclusions.

\section{Acknowledgments}

We acknowledge and appreciate our colleagues for their valuable efforts and comments on this paper.

\section{Statement of Ethics}

All animal experiments in this study were performed in accordance with the principles of the Animal Care Committee at China-Japan Friendship Hospital.

\section{Disclosure Statement}

The authors declare that they have no competing interests.

\section{References}

1 Sharawy N, Lehmann C. New directions for sepsis and septic shock research. J Surg Res. 2015 Apr;194(2): $520-7$.

2 Liu SF, Malik AB. NF-kappa B activation as a pathological mechanism of septic shock and inflammation. Am J Physiol Lung Cell Mol Physiol. 2006 Apr;290(4):L622-45.

3 Huet O, Chin-Dusting JP. Septic shock: desperately seeking treatment. Clin Sci (Lond). 2014 Jan;126(1):31-9.

4 Chen Y, Wang L, Kang Q, Zhang X, Yu G, Wan X, et al. Heat Shock Protein A12B Protects Vascular Endothelial Cells Against Sepsis-Induced Acute Lung Injury in Mice. Cell Physiol Biochem. 2017;42(1):156-68.

5 Seymour CW, Rosengart MR. Septic Shock: Advances in Diagnosis and Treatment. JAMA. 2015 Aug;314(7): 708-17.

6 Goodwin AJ, Guo C, Cook JA, Wolf B, Halushka PV, Fan H. Plasma levels of microRNA are altered with the development of shock in human sepsis: an observational study. Crit Care. 2015 Dec;19(1):440.

7 Rajasekaran S, Pattarayan D, Rajaguru P, Sudhakar Gandhi PS, Thimmulappa RK. MicroRNA Regulation of Acute Lung Injury and Acute Respiratory Distress Syndrome. J Cell Physiol. 2016 Oct;231(10):2097-106.

8 Martin GS. Sepsis, severe sepsis and septic shock: changes in incidence, pathogens and outcomes. Expert Rev Anti Infect Ther. 2012 Jun;10(6):701-6.

9 Huang RS, Hu GQ, Lin B, Lin ZY, Sun CC. MicroRNA-155 silencing enhances inflammatory response and lipid uptake in oxidized low-density lipoprotein-stimulated human THP-1 macrophages. J Investig Med. 2010 Dec; 58(8):961-7.

10 Xiang J, Bian C, Wang H, Huang S, Wu D. MiR-203 down-regulates Rap1A and suppresses cell proliferation, adhesion and invasion in prostate cancer. J Exp Clin Cancer Res. 2015 Jan;34(1):8.

11 Chi Y, Jin Q, Liu X, Xu L, He X, Shen Y, et al. miR-203 inhibits cell proliferation, invasion, and migration of nonsmall-cell lung cancer by downregulating RGS17. Cancer Sci. 2017 Dec;108(12):2366-72.

$12 \mathrm{Hu}$ YW, Wu SG, Zhao JJ, Ma X, Lu JB, Xiu JC, et al. VNN1 promotes atherosclerosis progression in apoE-/- mice fed a high-fat/high-cholesterol diet. J Lipid Res. 2016 Aug;57(8):1398-411. 
Ling et al.: Role of miR-203 in Septic Shock

13 Chai CY, Zhang Y, Song J, Lin SC, Sun S, Chang IW. VNN1 overexpression is associated with poor response to preoperative chemoradiotherapy and adverse prognosis in patients with rectal cancers. Am J Transl Res. 2016 Oct; 8(10):4455-63.

14 Jang HS, Kim J, Kim KY, Kim JI, Cho MH, Park KM. Previous ischemia and reperfusion injury results in resistance of the kidney against subsequent ischemia and reperfusion insult in mice; a role for the Akt signal pathway. Nephrol Dial Transplant. 2012 Oct;27(10):3762-70.

15 Chen S, Zhang W, Tang C, Tang X, Liu L, Liu C. Vanin-1 is a key activator for hepatic gluconeogenesis. Diabetes. 2014 Jun;63(6):2073-85.

16 Qu JQ, Yi HM, Ye X, Zhu JF, Yi H, Li LN, et al. MiRNA-203 Reduces Nasopharyngeal Carcinoma Radioresistance by Targeting IL8/AKT Signaling. Mol Cancer Ther. 2015 Nov;14(11):2653-64.

17 Yan X, Li W, Yang L, Dong W, Chen W, Mao Y, et al. MiR-135a Protects Vascular Endothelial Cells Against Ventilator-Induced Lung Injury by Inhibiting PHLPP2 to Activate PI3K/Akt Pathway. Cell Physiol Biochem. 2018; 48(3):1245-58.

18 Bardou P, Mariette J, Escudié F, Djemiel C, Klopp C. jvenn: an interactive Venn diagram viewer. BMC Bioinformatics. 2014 Aug; 15(1):293.

19 Shannon P, Markiel A, Ozier O, Baliga NS, Wang JT, Ramage D, et al. Cytoscape: a software environment for integrated models of biomolecular interaction networks. Genome Res. 2003 Nov;13(11):2498-504.

20 Li H, Wang S, Zhan B, He W, Chu L, Qiu D, et al. Therapeutic effect of Schistosoma japonicum cystatin on bacterial sepsis in mice. Parasit Vectors. 2017 May;10(1):222.

21 Collin SP. Topographic organization of the ganglion cell layer and intraocular vascularization in the retinae of two reef teleosts. Vision Res. 1989;29(7):765-75.

22 Heinemann AS, Pirr S, Fehlhaber B, Mellinger L, Burgmann J, Busse M, et al. In neonates S100A8/S100A9 alarmins prevent the expansion of a specific inflammatory monocyte population promoting septic shock. FASEB J. 2017 Mar;31(3):1153-64.

23 Takahashi G, Hoshikawa K, Matsumoto N, Shozushima T, Onodera C, Kan S, et al. Changes in serum S100A12 and sRAGE associated with improvement of the $\mathrm{PaO}(2) / \mathrm{FiO}(2)$ ratio following PMX-DHP therapy for postoperative septic shock. Eur Surg Res. 2011;47(3):135-40.

24 Agarwal V, Bell GW, Nam JW, Bartel DP. Predicting effective microRNA target sites in mammalian mRNAs. eLife. 2015 Aug; 4:4.

25 Mazloomi E, Jazani NH, Sohrabpour M, Ilkhanizadeh B, Shahabi S. Synergistic effects of glutamine and ciprofloxacin in reduction of Pseudomonas aeruginosa-induced septic shock severity. Int Immunopharmacol. 2011 Dec;11(12):2214-9.

26 Angus DC, van der Poll T. Severe sepsis and septic shock. N Engl J Med. 2013 Aug;369(9):840-51.

27 Chen Y, Wang G, Liu Z, Wang S, Wang Y. Glucocorticoids regulate the proliferation of T cells via miRNA-155 in septic shock. Exp Ther Med. 2016 Dec;12(6):3723-8.

28 Wang S, Zhao X, Wang J, Wen Y, Zhang L, Wang D, et al. Upregulation of microRNA-203 is associated with advanced tumor progression and poor prognosis in epithelial ovarian cancer. Med Oncol. 2013;30(3):681.

29 Latre de Late P, El Wakil A, Jarjat M, de Krijger RR, Heckert LL, Naquet P, et al. Vanin-1 inactivation antagonizes the development of adrenocortical neoplasia in Sf-1 transgenic mice. Endocrinology. 2014 Jul;155(7):2349_ 54 .

30 Zhang H, Sun T, Liu Z, Zhang J, Wang X, Liu J. Systemic inflammatory responses and lung injury following hip fracture surgery increases susceptibility to infection in aged rats. Mediators Inflamm. 2013;2013:536435.

31 Wang WC, Kuo CY, Tzang BS, Chen HM, Kao SH. IL-6 augmented motility of airway epithelial cell BEAS-2B via Akt/GSK-3 $\beta$ signaling pathway. J Cell Biochem. 2012 Nov;113(11):3567-75.

32 Wei J, Huang X, Zhang Z, Jia W, Zhao Z, Zhang Y, et al. MyD88 as a target of microRNA-203 in regulation of lipopolysaccharide or Bacille Calmette-Guerin induced inflammatory response of macrophage RAW264.7 cells. Mol Immunol. 2013 Oct;55(3-4):303-9.

33 Wang Z, Chi X, Liu L, Wang Y, Mei X, Yang Y, et al. Long noncoding RNA maternally expressed gene 3 knockdown alleviates lipopolysaccharide-induced inflammatory injury by up-regulation of miR-203 in ATDC5 cells. Biomed Pharmacother. 2018 Apr; 100:240-9.

$34 \mathrm{Yu} \mathrm{JB}$, Yao SL. Effect of heme oxygenase-endogenous carbon monoxide on mortality during septic shock in rats. Ir J Med Sci. 2009 Dec;178(4):491-6.

35 Li Y, Tong L, Zhang J, Zhang Y, Zhang F. Galangin Alleviates Liver Ischemia-Reperfusion Injury in a Rat Model by Mediating the PI3K/AKT Pathway. Cell Physiol Biochem. 2018;51(3):1354-63.

36 Yang F, Pei R, Zhang Z, Liao J, Yu W, Qiao N, et al. Copper induces oxidative stress and apoptosis through mitochondria-mediated pathway in chicken hepatocytes. Toxicol In Vitro. 2019 Feb;54:310-6.

37 Geng J, Xu H, Yu X, Xu G, Cao H, Lin G, et al. Rosuvastatin protects against oxidized low-density lipoproteininduced endothelial cell injury of atherosclerosis in vitro. Mol Med Rep. 2019 Jan;19(1):432-440.

38 Li P, Guo Y, Bledsoe G, Yang Z, Chao L, Chao J. Kallistatin induces breast cancer cell apoptosis and autophagy by modulating Wnt signaling and microRNA synthesis. Exp Cell Res. 2016 Jan;340(2):305-14.

39 Lu ZJ, Zhou Y, Song Q, Qin Z, Zhang H, Zhou YJ, et al. Periplocin inhibits growth of lung cancer in vitro and in vivo by blocking AKT/ERK signaling pathways. Cell Physiol Biochem. 2010;26(4-5):609-18. 